Wir haben bei diesen Kindern den Eindruck gewonnen, daß sie nach Uberstehen der Pleuritis keineswegs besonders ungünstigen äußeren Verhältnissen oder Reinfektionen ausgesetzt waren: Wir müssen also die Lungentuberkulose mit dem damals bereits vorhandenen Tuberkuloseinfekt in Beziehung bringen. Bei einem dritten Kinde sind zwar die Lungen von Tuberkulose freigeblieben, aber die Tuberkulose hatte sich an anderer Stelle lokalisiert: das I 3 jährige Kind kam I Jahr nach Uberstehen seiner Pleuritis wegen einer tubertulösen Wirbelkaries wieder in Behandlung der Klinik.

Bei.. zwei weiteren Fällen wurde nach einem Zeitraum von Io und II Jahren ein für Tuberkulose zweifelhafter Befund (leichte .Schallverkürzung über einer Spitze mit Helligkeitsunterschieden bei der Durchleuchtung) erhoben, in einem
Bei jenen 5 Kindern, die zur Zeit ihrer Erkrankung einen negativen Pirquet hatten, war eine Tuberkulose bei der Nachuntersuchung nicht festzustellen.

Unsere Nachforschungen über die Folgen der serösen idiopathischen Pleuritis haben somit ergeben, daß sie auch beim tuberkuloseinfizierten $K$ inde keine völlig harmlose Erkrankung ist; allerdings gestattet sie beim Kinde eine weit bessere Prognose gegenüber einer folgenden Tuberkulose als beim Erwachsenen.

Wir wollen uns mit der Feststellung dieser Tatsache begnügen und diese Unterschiede der beiden Altersstufen nicht mit. Hypothesen zu erklären versuchen. Den Angehörigen brauchen wir wegen der Zukunft des Kindes lreinesfalls Sorge ins Haus zu tragen. Es erscheint notwendig, dies zu betonen,

\begin{tabular}{|c|c|c|c|c|c|c|c|}
\hline Kljnik & $\begin{array}{c}\text { Zahl der nachuntersuchten } \\
\text { Kinder }\end{array}$ & Gesund & $\begin{array}{l}\text { Davon mit Resten } \\
\text { früherer Pleuritis }\end{array}$ & $\begin{array}{l}\text { Verdacht auf } \\
\text { Tbc pulm. }\end{array}$ & $\begin{array}{l}\text { Sichere Tbc } \\
\text { pulm. }\end{array}$ & $\begin{array}{l}\text { Tbc anderer } \\
\text { Organe }\end{array}$ & $\begin{array}{c}\text { Die sichere Tbc nach } \\
\text { wieviel Jahren festgestellt? }\end{array}$ \\
\hline $\begin{array}{l}\text { Berliner Univ,- } \\
\text { Kinder-Klinik }\end{array}$ & (24 Pirquet,+ 5 P. - ) & 23 & $(7)$ & 2 & $\begin{array}{c}3 \\
\text { (2 aktive Tbc } \\
\text { I ausgeh.Tbc) }\end{array}$ & $\begin{array}{c}\text { I } \\
\text { (Wirbel- } \\
\text { karies) }\end{array}$ & 3,2, I Jahren \\
\hline Wiener Univ.- & 39 & $3 \mathrm{I}$ & ( $\left.\mathrm{I}_{5}\right)$ & 4 & $\begin{array}{c}6 \text { oder rund } \\
4\end{array}$ & - & $\mathrm{I}^{1 / 4}, 7^{1 / 4}, 8, \mathrm{I}_{4}^{1 / 2} \mathrm{Jhr}$. \\
\hline Kinder-Klinik . : & (3I P.,+ 8 P. unbek.) & & - & & 8 oder rund & & \\
\hline $\begin{array}{l}\text { Baginskys Krank.- } \\
\text { haus Berlin }\end{array}$ & (P, unbekannt) & 29 & (9) & 2 & 2 & $\begin{array}{c}I \\
\text { (Coxitis) }\end{array}$ & $10,5^{1 / 2}, 7$ Jahren \\
\hline $\begin{array}{l}\text { Köster Kranken- } \\
\text { häuser Gothen- } \\
\text { burg in Schweden }\end{array}$ & (P. unbekannt) & $7 \mathrm{I}$ & (?) & $?$ & $\frac{5 \text { oder rund } 1}{? \text { oder rund } 1}$ & $?$ & zwischen I u. ro Jhr. \\
\hline
\end{tabular}

dritten Falle eine ausgeheilte Tuberkulose in Form dreier kleiner verkalkter Herde im rechten unteren Lungenfeld festgestellt. Diese drei befanden sich in gutem Ernährungszustand und fühlten sich wohl, wir hatten also weder subjektiv noch objektiv einen Anhaltspunkt für eine bestehende Lungenerkrankung.

Als Resultat unserer Beobachtungen ergibt sich demnach: von 24 Kindern, die zur Zeit ihrer Erkrankung an Pleuritis tuberkuloseinfiziert waren, blieben I8 nach $\mathrm{I}$ - Io Jahren völlig gesund, bei 7 von diesen waren nur geringe Reste der früheren Krankheit nachweisbar; 2 boten nach 2-3 Jahren sichere Zeichen einer aktiven, I einer überstandenen Lungentuberkulose. I Kind litt an einer tuberluulösen Wirbelkaries, 2 zeigten bei der Nachuntersuchung einen für Tuberkulose zweifelhaften, für ihre Träger derzeit jedoch völlig harmlosen Lungenbefund.

Unsere Ergebnisse stimmen mit denen anderer Kliniken und Krankenhäuser gut überein (s. Tabelle).

NoBEL und STEINEBACH ${ }^{1}$ ) sahen bei ihrem sehr genau geprüftem Material nach I-I9 Jahren unter 39 Fällen nur 4 mal deutliche Zeichen eines bestehenden tuberkulösen Lungenprozeses (nach I4 Monaten, $7^{1} / 2,8 \mathrm{bzw}$. $14 \frac{1}{1} / 2$ Jahren). 16 zeigten völlige Ausheilung, I 5 nur sehr geringe Andeutung an die frühere Krankheit, 4 einen für Tuberkulose zweifelhaften Befund. Unsere Ergebnisse decken sich auch mit den Erfahrungen Nathans ${ }^{1}$ ), der 34 Kinder aus dem. Krankenhaus BaginskYs I- io Jahre nach ihrer. Erkrankung wiedersah. Nur bei 2 Patienten ergab die Untersuchung das Vorhandensein tuberkulöser Lungenprozesse (Io und $5^{1 / 2}$ Jahre nach der Pleuritis), bei zweien lag Tuberkuloseverdacht vor, ein 3. Kind erkrankte nach 7 Jahren an einer tuberkulösen Coxitis. Ich möchte noch besonders auf die Ergebnisse der Arbeit Köstens ${ }^{1}$ ) hinweisen, weil er an Hand eines großen Erwachsenen- und Kindermaterials vergleichende Untersuchungen über die Prognose der serösen idiopathischen Pleuritis in den beiden Lebensaltern anstellen konnte. Während eine Lungentuberkulose, wie eingangs schon erwähnt, bei Erwachsenen, die älter als I 5 Jahre waren, in mindestens der Hälfte aller Fälle auftrat, war die Prognose bei Kindern unter I5 Jahren weit besser: Inmerhin zeigte doch $1 / 3$ aller von KöSTER beobachteten Kinder später tuberkulöse Veränderungen der Lungen.

1) 1. c. denn mehrmals war dem Kinde nur eine Lebensdauer von höchstens Io $-\mathrm{I}_{5}$ Jahren von dem behandelnden Arzte prophezeit worden. Andererseits dürfen wir aber doch die Kinder nicht ganz aus dem Auge verlieren. Beginnende Lungenerkrankungen werden dann, wie bei einem unserer Fälle, frühzeitig genug erkannt werden, um einer wirksamen Therapie, wenn möglich der Pnẹumothoraxbehandlung, zugeführt zu werden $^{1}$ ).

\section{IST BEI PLACENTA PRAEVIA DIE METREURYSE EIN FÜR DIE HAUSÄRZTLICHE GEBURTSHILFE GEEIGNETES UNGEFÄHRLICHES VERFAHREN?}

\section{Von}

Prof. Dr. Walther Hannes, Oberarzt der Poliklinik. Aus der Breslauer Univ.-Frauenklinik. (D'rektor Geh. KÜSTNER.)

Da der im praktischen Berufsleben stehende Arzt heute unendlich schwieriger und seltener als noch vor dem Kriege Zeit für eigene praktische Fortbildung an entsprechenden Anstalten bzw. in derartigen Kursen erübrigen kann, so ist es nötig und mit Freude zu begrüßen, wenn ain weiten ärztlichen Kreisen zugänglicher Stelle von berufener Seite wichtige geburtshelferische Fragen für den Praktiker praktisch erörtert werden.

In diesem Sinne und mit dieser Absicht schrieb DöDERLEIN in Nr. 4 der Münch. med. Wochenschr. über das ja für den Praktiker so ungemein wichtige Kapitel „Placenta praevia“".

Da DöDERLEIN hierbei unter anderem zu dem Schlusse kommt, daß die Metreuryse kein für die hausärztliche Geburtshilfe geeignetes, ungefährliches Verfahren sei, so kann ich nicht umhin, da ja gerade von unserer Schule seit Jahrzehnten der Intrauterinballon als Behandlung der Placenta praevia in der hausärztlichen Praxis empfohlen wird, wieder einmal zu dieser Frage Stellung zu neimen.

1) Das Material ist ausführlich bearbeitet in einer Dissertation von JANCU. 
Nach seinen Ausführungen scheut DöDERLEIN die bei Einlegen des Ballons in die Amnionhöhle womöglich auftretende Blutung, die schon bei Berührung der Placenta mit dem Pol des Ballons eine sehr umfängliche, ja gefährliche werden kann. Bezüglich der Ausführung der kombinierten Wendung in diesen Fällen scheint - da hierüber nichts in dem Aufsatz steht - DöDERLEIN solche Blutungen nicht zu fürchten. Diese ganze Deduktion scheint mir theoretisch und praktisch nicht recht verständlich.

Nehmen wir an, es handelt sich um den üblichen Fall von Placenta praevia totalis mit Blutung und für zwei Finger durchgängigem Muttermund und Halskanal bei einer Mehrgebärenden. Notwendig ist zwecks sachgemäßer Blutstillung die Tamponade vom Einnern her, wobei der Tampon von oben den gelösten Placentarbezirk auf seinen ursprünglichen Haftbezirk so fest aufdrückt, daß dabei die vorher bei $\mathrm{Ab}$ lösung dieses P̉lacentarteiles eröffneten Uteroplacentargefäße nicht mehr bluten können. Dann sind sie richtig durch Tamponade verschlossen. Es besteht heute nirgends mehr Zweifel oder Diskussion darüber, daß dieses Ziel erreicht werden kann, sowohl durch den nach Ausführung der kombinierten (BRAXTON-Hicksschen) Wendung auf die Placenta herabgezogenen und am herausgeleiteten FuB zwecks Fixation belasteten Stei $\beta$, als auch durch die intraamniale analog am Schlauch belastete Metreuryse. Auch DöDERLEIN schließt sich diesen Anschauungen auf Grund seiner Erfahrung an.

Ganz gleichgültig nun, ob in dem angenommenen Falle zur Erzielung korrekter Blutstillung der Geburtshelfer den Braxton-Hicks oder den Intrauterinballon benutzen will; es muß zunächst der vom vorliegenden Lappen der Placenta blockierte Weg ins Eiinnere freigemacht werden. Dies geschieht mittels Durchbohrung des vorliegenden Lappens Placentargewebes in situ entweder mit dem durchbohrenden Finger oder mittels eines unter Leitung des Fingers eingeführten stumpfen Instrumentes, am besten einer Kornzange oder breiten stumpfen Klemme. Der nachgeschobene Finger bzw. ein Spreizen des zum Durchbohren benutzten Instrumentes erweitern den Zugang zur Eihöhle in genügender Weise. Daß bəim stumpfen Durchbohren der Nachgeburt in so geschilderter Weise größere Gefäße des fötalen Kreislaufes im Chorion ganz typisch unverletzt zur Seite weichen, konnte bereits vor ca. 20 Jahren Wilhelm PoNfICK an Placenten so behandelter Fälle eindeutig demonstrieren.

Wird nun die kombinierte Wendung ausgeführt, so gehen nach Einführen der ganzex Hand in die Scheide zwei Finger per Placentam perforatam in die Eihöhle und suchen unter energischer Hilfe der anderen von außen wirkenden Hand einen $F u ß z u$ erfassen, der dann, falls zwei Finger bequem in die Gebärmutterhöhle eingeführt werden. konnten, unschwer herabgezogen und hierauf die Wendung.vollendet werden kann. Treffend schildert DöDERLEIN, wie schwer für den , nicht sehr geschulten" Geburtshelfer so eine Wendung bei nur für zwei Finger eingängiger Gebärmutter sich gestalten kann, wie sehr es darauf ankommt, die zwei Finger, die eingeführt werden können, möglichst hoch hinauf in die Eihöhle zu schieben, um die Erfassung des Fußes bewerkstelligen zu können. Je mehr - oft ist ja das Erfassen des Fußes nicht leicht, und oft $\mathrm{mu} \beta$ dieser Eingriff vom allgemeinen Praktiker mit nur gelegentlicher geburtshilflicher Übung ausgeführt werden - je mehr nun die Finger in die Höhe geschoben werden, um so mehr läuft man Gefahr, die durchbohrte Placenta gewissermaßen auf die durchgeführten Finger aufzuhülsen und somit in die Höhe zu schieben, d. h. sie mehr abzulösen und neue zu neuer Blutung neigende Uteroplacentargefäße zu eröffnen. Die Gefahr, daß es während der Ausführung der kombinierten Wendung blutet, wächst konform ihrer Schwere, d. h. je länger es bis zum Herabziehen des Fußes im Einzelfalle dauert, um so mehr kann und wird es dabei bluten.

Wie steht es nun bei Ausführung der intraamnialen Metreuryse? Der zigarrenförmig zusammengerollte weiche Braunsche Ballon, dessen alleinige Anwendung von uns immer empfohlen wird, wird in die mit einer Beckenkrümmung versehene kornzangenartige Zange so gefaßt, daß die
Ballonspitze eben gerade über das Zangenende übersteht und unter Leitung eines Fingers glatt und schnell durch.das zuvor geschaffene Loch im vorliegenden Lappen des Mutterkucheris in die Amnionhöhle eingeschoben. Der in besagter Weise zusammengefaltete und gefaßte Ballon entspricht in seinen Dimensionen etwa einem Finger, kann also bequem und ohne eine Dislokation an der Placenta hervorzurufen durch das' geschaffene Loch eingeschoben werden. Bei der nun folgenden Auffüllung des Ballons mit ca. $500-600 \mathrm{ccm}$ steriler Flüssigkeit entfaltet sich der von der Zange freigegebene Ballon auf der amnialen Fläche der Placenta, ohne irgendwie die Tendenz zu haben, den Mutterkuchen nach oben, d. h. im Sinne weiterer Ablösung zu dislozieren. Im Gegenteil, je mehr der Ballon durch die Auffüllung sich entfaltet, um so mehr beginnt er bereits, die Placenta von ihrer amnialen Fläche her, also vom Eiinnern aus, auf ihre regelrechte Unterlage an der Uterus- bzw. Cervixwand auf- und anzudrücken. Diese Tamponade von oben ist unverrückbar perfekt, sowie nach beendeter Auffüllung der Schlauch des Ballons mit $600-700 \mathrm{~g}$ belastet ist.

Es ist mithin nicht ganz ersichtlich, weshalb, wie DÖDERLEIN ausführt, es gelegentlich schon bei Berührung des Poles des Ballons mit der Placenta zu besonders abundanter und ,,sofort zu raschem Handeln" zwingender Blutung kommen soll, während er solche Blutung, die doch nur in neuerlicher $\mathrm{Ab}$ lösung der Placenta von ihrer Haftfläche begründet sein kann, bei Ausführung einer technisch schwierigen kombinierten Wendung selbst von geburtshelferisch wenig geschulter Hand nicht zu fürchten scheint.

Ich kann mir die von DöDERLEIN bei seinen 6 Metreurysenfällen bei Placenta praevia beobachteten Blutungszwischenfälle nur erklären durch ein zu breites Überstehen des Ballors über die Haltezange. Dies ist möglich, wenn nicht ein BRAUNscher weicher birnförmiger Ballon, sondern ein kegelförmiger sog. zugfester Ballon mit unnachgiebiger breitüberstehender Basis verwandt wird. Aus welchen anderen Gründen ich auch sonst den weichen Ballon dem zugfesten vorziehe, habe ich a. a. O. verschiedentlich auseinandergesetzt.

Nun stehen wir alle auf Grund unserer praktischen Erfahrungen und gestützt auf diesbezügliche klinische $Z_{u}$ sammenstellungen von ZWEIFEL, LIEGNER und anderen auf dem Standpunkt, daß nach einmaligem Einsetzen der Blutung bei Placenta praevia gegen Ende der Schwangerschaft bzw. im Beginn der Geburt jeder weitere Blutverlust verhängnisvoll werden kann und somit abzuwenden bzw. durch Zurechthaltung sachgemäßer schnell - eingreifender und prompt wirkender Hilfe auf ein möglichstes Gefahrenminimum einzuengen ist. DöDERLEins neuerliche eindrückliche Mahnung an die Praktiker, solche Schwangere nach stattgehabter, zum Stehen gekommener Blutung nicht einfach zuwartend ins Bett zu legen, sondern, falls sie nicht an Ort und Stelle sogleich entbunden werden können, schleunigst einer geburtshilflichen Anstalt zuzuführen; ist unbedingt $z u$ begrüßen und $z u$ unterstützen.

Ofters liegt nun bei Blutung sub partu infolge Placenta praevia der Fall nicht so, daß die Gebärmutter für zwei Finger eingängig ist, sondern nur eben für einen Finger, dann ist es für den allgemeinen Praktiker mit nicht ganz besonders geburtshilflicher Ubung so gut wie ausgeschlossen, die kombinierte Wendung auszuführen. JoLLY und sehr bald nach ihm HENKEL empfahlen für solche Fälle vor ca. I5 Jahren dem geübten Geburtshelfer mit einer scharfen (JoLLY) oder einer stumpfen (HENKEL) Zange den in den Bereich des einen eingeführten Fingers gebrachten $F u B$ zu erfassen und ihn in Spitzfußstellung durch den engen Cervicalkanal und Muttermund durchzuleiten. DöDERLEIN empfiehlt jetzt dem Praktiker dieses Verfahren für die nichtleichten Fälle von Braxton'Hicks bei nur eben für zwei Finger durchgängigem Ausführungsgang der Gebärmutter, wo die Durchleitung des gefaßten Fußes mit zwei Fingern auf Widerstand stößt. Es will mir nicht einleuchten, daß solche schwierige kombinierte Wendungen, wo mehrfaches bis zu ibrem Gelingen versucht, wo mehrfach sicherlich mit Hand bzw. den zwei Fingern die perforierte, ihnen dicht anliegende Placenta hin und her 
passiert wird, ablaufen ohne stärkere Blutungen. Jedenfalls sprechen unsere Erfahrungen dafür, daß in solchen Fällen die kombinierte Wendung keineswegs für die durch Blutung gefährdete Kreißende eine blutsparende Operation ist.

Ganz anders die intraamniale Metreuryse! Sie ist mit dem weichen BRAunschen Ballon auch für den nur über Durchschnittserfahrung in der Geburtshilfe verfügenden Praktiker ausführbar, wenn der Muttermund eben für einen Finger durchgängig ist, also viel eher als die kombinierte Wendung. Mit in Kauf $\mathrm{zu}$ nehmen ist die meist nicht beträchtliche Blutung bei der zwecks Ausführung der Manipulation zunächst bei totaler Placenta praevia vorzunehmenden Durchbohrung der Placenta. Hier ist nach unseren Erfahrungen ein stumpfes Vorgehen mit Finger bzw. Kornzange zweckmäßiger als ein scharfes mit dem Troikart, weil dem stumpfen Instrument erfahrungsgemäß die größeren kindlichen Gefäße der fötalen Placentarfläche besser ausweichen, als einem scharfen. Zudem wird man bei Anwendung des Troikarts oft zwei Handgriffe vornehmen müssen, weil das Troikartloch noch stumpf bis auf die nötige Lichtung erweitert werden muß.

Immer noch wird auch gelegentlich der Metreuryse als Nachteil angerechnet, daß sie ein eigenes Instrumentarium benötige und daß gelegentlich im Moment des Gebrauches ein Ballon defekt werden kann. So empfiehlt STöckel in seinem Lehrbuch, weil dem Praktiker diese Methode nicht einfach genug sei und er ihre technischen Schwierigkeiten überschätze, diese nur anzuwenden; wo die kombinierte Wendung noch nicht möglich ist, denn die bereits bei für einen Finger durchgängigem. Muttermund mögliche Netreuryse sei eigentlich fast immer schon nach der ersten Blutung ausführbar.

Obwohl also zugegeben wird, daß die Methode des Intrauterinballons für das Gros der in Rede stehenden Fälle sachlich am Platze ist, soll sie doch nicht immer ausgeführt werden. Daß, wie STöckEI meint, aseptische Fehler beim Sterilisieren und Einführen vorkommen können, ist nichts für die Metreuryse Typisches; wenn wir solche Argumente heranziehen, dann müssen alle Encheiresen in der Geburtshilfe seitens des allgemeinen Praktikers in dieser Hinsicht der Beargwöhnung unterliegen. Weiter bemängelt STÖcKEL die Sicherheit der Blutstillung durch den Ballon; unsere große Erfahrung hier kann jegliche Zweifel in dieser Hinsicht zerstreuen; auch der weiche Braunsche Ballon stillt, intraamnial eingelegt, sicher die Blutung, sofern die Belastung richtig ist (bei $500 \mathrm{ccm}$ Füllung etwa $600-700 \mathrm{~g}$ Belastung). Ist die Belastung zu gering, dann ist die Tamponadewirkung zu gering, ist sie zu stark, dann zieht sich der Ballon wurstförmig und ohne seine Aufgabe (Blutstillung und Erweiterung durch Anregung regelrechter Wehentätigkeit) erfüllt zu haben, durch den Halskanal und Muttermund durch. Und diesen Mangel der Abhängigkeit in Wirkung und Erfolg von der Art der Belastung hat auch die kombinierte Wendung nur mit dem verhängnisvollen Unterschied, daß bei zu starker Belastung bzw. Zug am herausgeleiteten Fuß nur zu oft tödliche Cervixrisse die Folge sind, während der wurstförmig sich durchziehende weiche Ballon in dieser Hinsicht ungefährlich ist.

LIEPMANN charakterisiert meines Erachtens treffend in seinem Geburtshilfe-Seminar die Situation des allgemeinen Praktikers mit folgendem Bericht aus seiner geburtshilflich poliklinischen Tätigkeit: „Ich habe niemals von einem in der Geburtshilfe nur wenig erfahrenen Arzt die Braxton-HicksWendung bei Placenta praevia gelingen sehen, wohl aber die Metreuryse. Und ich stehe auch nicht an, zu behaupten, daß die Metreuryse für den Anfänger leichter auszuführen ist als die Wendung nach Braxton-Hrcks."

Die Metreuryse muß eben in unseren geburtshilflichen Operationskursen ebenso gelehrt werden wie Zange und Wendung. Und das hierzu nötige Instrumentarium muß ebenso bereit und instand gehalten werden seitens des Geburtshilfe ausübenden Arztes wie z. B. das CraniotomieInstrumentarium, das ja auch nur selten von ihm benötigt wird.

Wir sind uns wohl alle einig, daß eine mit Placenta praevia behaftete Kreißende vom Arzt nie vor Abschluß der Nach- geburtsperiode verlassen werden soll; hiermit fällt auch die letzte immer wieder und auch von STÖcKEL in seinem Lehrbuch gegen den Intrauterinballon erhobene Einwendung in sich zusammen, daß nach Geburt des Ballons meist noch ein zweiter Eingriff, gewöhnlich die innere Wendung, nötig sei. Da ja der Arzt anwesend bleiben muß, so kann hieraus kein Nachteil für die ihm anvertraute Kreißende erwachsen. Kündigt das Vorrücken des Ballons seine bevorstehende Geburt an, so wird die Belastung entfernt, bis der Geburtshelfer sich $\mathrm{zu}$ einem von neuem nötig werdenden Eingriff desinfiziert hat. Dann wird der Ballon mit leichtem Zug, sowie er in die Scheide geboren ist, entfernt; beginnt es jetzt wieder zu bluten bzw. besteht eine operative Entbindung indizierende Kindeslage, so wird nunmehr bei genügend erweiterten mütterlichen Weichteilen unschwer und rasch die im vorliegenden Fall nötige entbindende Operation ausgeführt werden können. Außerdem muß ja auch beim BraxtonHicks sich der Arzt instand halten, um nochmals manuell bei Arm- und Kopfentwickelung einzugreifen.

Aus allen vorstehenden Ausführungen geht wohl hervor, daß die Gründe, die von den Anhängern der kombinierten Wendung nach BRAxroN-HICKs gegen die intraamniale Metreuryse ins Feld geführt werden, auch für den geburtshilflichen Praktiker nicht stichhaltig sein können.

Rechtfertigen nun die Resultate für Mutter und Kind die Empfehlung des Intrauterinballons auch für die breite Praxis? Es gibt wohl heute kaum noch einen an maßgeblicher Stelle befindlichen Geburtshelfer in Deutschland, der nicht zugäbe, daß ungleich viel mehr Kinder am Leben erhalten werden bei der Placenta praevia mittels Metreuryse als mittels Braxton-Hicks.

DöDERLErN, dessen jüngste Ausführung ja der Anlaß zu dieser Auseinandersetzung sind, legt Gewicht darauf, seine Resultate ohne jegliche Abzüge von bei Einlieferung der Mutter bereits abgestorbenen oder lebensschwachen und lebensunfähigen Kindern zu bringen.

Er berichtet über 50 mit kombinierter Wendung behandelte Fälle von Placenta praevia, von denen 5 Mütter und 24 Kinder starben. Dieses gibt eine mütterliche Mortalität von $10 \%$ und eine kindliche von $48 \%$. Gleichzeitig zitiert DöDERLEIN eine Sammelstatistik von i 266 klinisch mittels Braxton-Hicks behandelte Praeviafälle. Hier betrug die mütterliche Mortalität $5,45 \%$ und die kindliche $79.3 \%$.

Hier zeigt sich also wieder das für die Braxton-HicksMethode so klassische Verhältnis: je mehr lebende Kinder, um so mehr tote Mütter und je mehr tote Kinder, um so mehr lebende Mütter! Diese Methode stellt eben dem Temperament des behandelnden Arztes so manche Schlinge; so kommt es doch einmal $\mathrm{zu}$ einer voreiligen, vorzeitigen und der Kreißenden verhängnisvoll werdenden Extraktion, zu der es nie gekommen wäre, wenn nicht durch die Wendung eine unvollkommene Fußlage hergestellt worden wäre.

Um auch meinerseits Zahlen ohne jeden Abzug zu bringen, verweise ich auf meine Publikation in Med. Klinik 1908, Nr. 28 und entnehme ihr folgende Zahlen: Von II9 (50 auf der stationären Klinik und 69 in der Poliklinik) mittels Metreuryse wegen Placenta praevia behandelten Frauen gingen 6 i. e. $5 \%$ zugrunde. Diese I I9 Frauen brachten 6I $(5 \mathrm{1}, 2 \%$ ) lebende Kinder zur Welt. Nebenbei sei nur bemerkt, daB I3 Kinder bei Beginn der Behandlung bereits abgestorben waren.

Recht bedeutsam scheinen mir für unserer Frage auch die von DöDERLEIN in den Fällen erzielten Erfolge, wo sogleich an die Wendung da der Muttermund genügend erweitert war, die Extraktion angeschlossen werden konnte. Hier verzeichnet er $25 \mathrm{Fälle}$ mit 3 i. e. I $2 \%$ toten Müttern und I 3 i. e. $54 \%$ toten Kindern. Hierzu entnehme ich Zahlen aus meiner Publikation im Zentralbl. f. Grynäkol. I 909 und berichte, daß bei uns in 38 Fällen sogleich an die Wendung die Extraktion angeschlossen werden konnte mit 3 i. e. $7,8 \%$ toten Müttern und i 8 i. e. $47 \%$ toten Kindern.

Der nämlichen Publikation entnehme ich bezüglich unserer Erfolge mit Braxton-Hicks folgende Zahlen. Unter 26 Fällen von kombinierter Wendung haben wir eine kindliche Mortalität von $92 \%$ (24 Fälle) und eine mütterliche von $3,0 \%$ (I Fall) 2 u verzeichnen.

Diese wenigen Zahlen beweisen meines Erachtens schon ganz eindeutig die schon seit jeher von allen Seiten als 
Leitsatz für die Placenta praevia-Behandlung in der breiten Praxis aufgestellte Forderung als gerecht, daß dem in der allgemeinen Praxis stehenden Geburtshelfer nur solche Verfahren zur Besserung der hier bisher so ganz besonders schlechten Resultate der Kinder empfohlen werden dürfen, durch die die Resultate für die Mütter nicht verschlechtert werden. Daß dies nicht durch die Empfehlung und Verbreitung der kombinierten Wendung, wohl aber durch die intraamniale Metreuryse zu erreichen ist, zeigen die obigen Zahlen und vorstehenden Ausführungen. Es gibt keinen wirklich stichhaltigen und ernsthaft zu diskutierenden Grund, der gegen die Erlernung und den Gebrauch des Intrauterinballons in der nunmehr abermals von mir genügsam erläuterten Weise bei der Placenta praevia seitens des geburtshilflichen Praktikers spräche. Wer sie kann, wird auch nur äußerst selten draußen in der Praxis in die Verlegenheit kommen die Scheidentamponade zu machen, weil die intraovuläre Tamponade noch nicht ausführbar ist. DaB solche Scheidentamponade, wenn sie auch noch so exakt und unter Wahrung aller aseptischen Kautelen ausgeführt wird, doch eben nur einen "augenblicklichen Notbehelf" darstellen darf, hat ja DöDERLEIN wiederum klassisch geschildert.

Eine ganz besondere Aufmerksamkeit ist bei Abwartung der Geburt bei Placenta praevia der Nachgeburtsperiode zuzuwenden; hier gibt es bei Lösung der ja oft zum Teil in der Cervix sitzenden Placenta Störungen, und da ja jedwede Störung im Ablauf einer Nachgeburtsperiode so gut wie gleichbedeutend ist mit vermehrtem Blutverlust in dieser Zeit, so droht von dieser Seite den bereits durch Blutung ante partum geschädigten Frauen neues Verhängnis. Jeder kleinste anormale Blutverlust post partum kann gelegentlich, wie die Erfahrung lehrt, den Tod bedingen. So empfiehlt DöDERLEIN sofort post partum die Placenta mittels CREDÉschen Handgriffes herauszudrücken oder sie manuell herauszuholen und sogleich eine feste Uterovaginaltamponade nach DüHrssen anzuschließen. Störungen in der Nachgeburtsperiode und namentlich Blutungen ex atonia waren es ja, die Sellieim und andere veranlaßten, jede Geburtsdehnung des mit zum Placentarbezirk einbezogenen Ausführungsganges der Gebärmutter $\mathrm{zu}$ scheuen und somit für möglichst frühzeitigen cervicalen Kaiserschnitt bei Placenta praevia einzutreten.

Bereits auf dem Kongre $\beta$ zu Straßburg 1909 wies ich darauf hin, da $\beta$ bei uns die von allen Seiten so gefürchteten Störungen in der Nachgeburtsperiode bei Placenta praevia zu den Seltenheiten gehörten, und daß dies meines Erachtens als Folge des Intrauterinballons anzusehen sei, indem eben die durch den Ballon in physiologische Bahnen gelenkte Wehentätigkeit, die ja bekanntlich primär bei Placenta praevia meist viel zu wünschen übrig läßt, auch nun in der Nachgeburtsperiode zu weiterer physiologischer Auswirkung kommt. In den geb. Lehrbüchern und in klinischen diesbezüglichen Statistiken (z. B. DORANTH, Wien) findet man Io bis I $2 \%$ manuelle Placentarlösungen bei Placenta praevia angegeben. Heinz Küstner hat diesbezüglich unser Material in letzter Zeit bearbeitet und gefunden, daB bei 23I mit Metreuryse behandelten Fällen von Placenta praevia im ganzen 6 manuelle Lösungen i.e. $2,6 \%$ und $8 \mathrm{mal}$ i. e. $3,5 \%$ noch die manuelle Entfernung zurückgehaltener, nur ganz lose in der Gegend des inneren Muttermundes sitzender Cotyledonen nötig war; $29 \mathrm{mal}$ (12,5\%) wurde mittels Expression nach Credé behandelt. In keinem Falle ist eine Gebärmutterscheidentamponade nötig gewesen.

Auf Grund unserer nicht kleinen klinischen und poliklinischen Erfahrungen können wir also die intraamniale Metreuryse dem Praktiker besonders auch von dem Gesichtspunkt aus empfehlen, da $\beta$ dann die Nachgeburtsperiode unter Anwendung der auch sonst ihm üblichen und gebräuchlichen Hilfsmittel und Methoden günstig für die Mutter ablaufen wird.

Die Erfahrungen unserer Schule würde ich für den Praktiker folgendermaßen zusammenfassen:

r. Treten infolge Placenta praevia Blutungen auf, so sind solche Frauen, wenn ohne Gefahr für sie möglich, einer Gebäranstalt zuzuführen. Ist dies nicht angängig, so $\mathrm{muß}$ alsbald an Ort und Stelle für sachgemäße Blutstillung Sorge getragen werden.

2. Dies geschieht, wenn der Muttermund und Halskanal wenigstens für einen Finger durchgängig ist, mittels intraamnialer Metreuryse, falls es sich um Placenta praevia totalis bzw. um Querlage handelt.

3. Sind neben dem Placentargewebe freie Eihäute im Muttermunde $\mathrm{zu}$ tasten und besteht Längslage der Frucht, dann kann zunächst versucht werden, mit dem Blasensprung auszukommen. Tritt hierauf nicht unter Aufhören der Blutung der vorliegende Teil tiefer, dann soll ebenfalls die Metreuryse eingeleitet werden.

4. Zur Metreuryse bei Placenta praevia ist der weiche nicht zugfeste BRAUnsche Ballon zu verwenden.

5. Nur zum $Z_{w e c k}$ des Transportes und nur wenn bei Behandlung an Ort und Stelle bei fortbestehender Blutung der Muttermund noch nicht für einen Finger durchgängig ist, darf, sozusagen als kurz dauernder Notverband, die peinlich aseptisch ausgeführte Scheidentamponade zur Anwendung kommen.

6. Ist bei Übernahme des Falles der Muttermund regelrecht für eine entbindende Operation eröffnet und besteht noch Blutung, dann ist die nach Lage und Stand des Kindes indizierte entbindende Operation auszuführen.

7. Die kombinierte Wendung nach BRAXTon-HICks ist nur dann bei Placenta praevia anzuwenden, wenn es sich um eine sicherlich lebensunfähige Abortusfrucht handelt, oder wenn aus irgendwelchen äußeren Gründen das Metreuryseinstrumentarium nicht zur Hand ist. Wird in solchem Falle vor völliger Eröffnung des Muttermundes an dem herausgeleiteten Fuß extrahiert, so sind Cervixrisse und tödliche Blutungen die sehr wahrscheinliche Folge.

Ich will diese Auseinandersetzung nicht schließen, ohne die Frage $z u$ streifen, welche ja auch für das Verhalten des Praktikers in manchem Falle leitend sein kann, ob der Kaiserschnitt imstande ist, die Resultate für Mutter und Kind bei Placenta praevia zu bessern. Ich möchte meinen, daß diese Frage für den ausgesuchten Einzelfall zu bejahen, für die große Masse der Fälle aber wohl zu verneinen ist. Werden mit Placenta praevia behaftete Kreißende, deren Früchte mindestens der 36 . bis 37 . Schwangerschaftswoche angehören und noch ungeschädigt sind, frühzeitig in aseptisch einwandfreiem Zustande (d.h. untamponiert und höchstens mit Gummihandschuhen bekleideter Hand untersucht) in die Gebäranstalt eingeliefert, dann halte ich den transperitonealen Kaiserschnitt für einen Eingriff, der das kindliche Leben mit nahezu absoluter Sicherheit rettet; ohne die Mutter mehr zu gefährden, als sie durch die Behaftung mit Placenta praevia an sich in ihrem Leben gefährdet ist. So wird meines Erachtens namentlich in solchen Fällen, wenn es sich um Erstgebärende handelt, der Kaiserschnitt segensreich sein. In irgendwie größerer und damit für die Gesamtresultate bei Placenta praevia in Betracht kommender Menge werden wir solche für den Kaiserschnitt geeigneten Fälle von Placenta praevia aber nur bekommen, wenn der Praktiker diese Frauen schon beim Eintritt der ersten, womöglich nur ganz schwachen und vorübergehenden Blutung mittels Krankenauto in eine Gebäranstalt überführen lassen wird.

\section{ÜBER DIE WIRKUNGSWEISE UND DAS ALTERN DER VACCINE. ${ }^{\text {) }}$}

\author{
Von \\ CARL LANGE. \\ Aus dim Laboratorium von Prof. CARL LANGE in Barlin.
}

Wenn wir uns veranlaßt sehen, die Arbeit von A. BuschKE und E. LANGER, mit deren Ausführungen wir übrigens bis auf geringe Differenzen übereinstimmen; durch einige Beobachtungen $z u$ ergänzen, so liegt das daran, daß $\beta$ wir auf Grund langjähriger

2) Vgl. die gleichlautende Veröfentlichung von A. BUSCHKE und E. LANGER in Nr. 3 , S. r2z dieser Zaitschrift. 